\title{
A New Optimal Method of Tool Path Generation for Slow Tool Servo Turning of Complex Surface
}

\author{
Hangyan Guo, Min Kang, Wei Zhou, Hengtai Niu, Bingwei Song \\ College of Engineering, Nanjing Agricultural University, No.40, Dianjiangtai Road, Pukou Distinct, Nanjing \\ 210031. China._Email: 2019112003@njau.edu.cn, kangmin@njau.edu.cn, 1506036510@qq.com, \\ 1551903163@qq.com,542508756@qq.com
}

In order to improve the surface machining quality of slow tool servo (STS) turning in complex surfaces, the optimal method of tool path generation (TPG) was studied. Taking into consideration the problem of large discrete errors and interpolation errors in TPG, equal height discretization method and interpolation algorithm for non-uniform nodes were proposd and the acceleration continuous condition was introduced. Simulation results showed that equal height discretization method could reduce the discrete error by more than $70 \%$. The interpolation errors could be reduced to two orders of magnitude by transforming segment cubic Hermite interpolation into segment cubic spline interpolation. Finally, the processing experiments were performed. The results showed that the form error PV value for the workpiece of the toric surface obtained by equal height discretization method and non-uniformity processing and segment cubic spline interpolation reached $0.002 \mathrm{~mm}$. The PV value of the sinusoidal array surface was about $0.009 \mathrm{~mm}$, and its surface roughness value was $0.064 \mu \mathrm{m}$. The results proved this method can effectively reduce the discrete errors and interpolation errors, as well as improve the surface machining quality.

Keywords: Tool path, Equal height discretization, Hermite interpolation, Non-uniformity processing, Spline interpolation

\section{Introduction}

Complicated curved surfaces (such as aspherical surfaces or free-form surfaces) can be used for certain types of optical components to make them have better optical characteristics in the optical system, obtain high-quality images, optimize system structure, and improve and expand system functions [1-3]. With the increasing application of complex curved surfaces in aerospace, astronomy and mechanical fields, ultra-precision machining of complex curved surfaces has become an important factor determining its development [4-8]. As an ultra-precision machining method, slow tool servo (STS) turning has been widely used in the machining of many different types of complex curved surfaces in recent years due to its high surface machining accuracy and machining efficiency.

In spite of the fact that a lot of research has been carried out for the STS turning of complex surfaces, the tool path generation (TPG) technique is still the key technology because it has a direct impact on the surface accuracy [9]. The overall scheme of TPG for STS turning of complex surfaces is as follows: First, the complex surfaces expressed by equation or other ways is discretized into cutting contact points (CCPs). Afterwards, discrete CCPs are translated into discrete cutting location points (CLPs) through tool geometry compensation. Then, trajectory interpolation for CLPs is completed. Finally, the verified
$\mathrm{NC}($ Numerical Control) program is exported for machining. The main routes of the TPG can be summarized as CLPs generation and CLPs trajectory interpolation. CLPs generation includes CCPs discretization and tool geometry compensation. Reasonable CCPs discretization method is the basis of TPG. For three axes turning of complex surfaces, spiral tool path is the most typical method [10].

At present, the equal angle discretization method and the equal arc length discretization method are the two main methods of CCPs discretization [11-16]. However, these two methods do not consider the height difference of every two adjacent CCPs, so the discrete error is relatively large. Nowadays, B-spline interpolation [17] and Non-Uniform Rational B-Splines (NURBS) interpolation $[12,18]$ are commonly used for CLPs interpolation, but the interpolation error is relatively large. Guan [10] and Wang et al. [9] proposed Hermite interpolation method, but this method cannot guarantee that the interpolation path is two order continuous, that is, the acceleration is discontinuous, which results in the interpolation path not being smooth enough. At the same time, the choice of interpolation node also affects the interpolation error to a greater extent, but most interpolation methods do not take this problem into consideration.

In view of the above-mentioned problems in the process of TPG, a systematical method of TPG for STS turning of complex surfaces was proposed. This 
method includes the generation of CCPs, selection of interpolation nodes and calculation of two order continuous interpolation. In this method, the height difference of every two adjacent CCPs was taken into consideration, the interpolation algorithm for non-uniform nodes, and the conversion method from Hermite interpolation to Spline interpolation were given. This ensured the two-order continuity of the interpolation path.

\section{Generation of cutting location points}

\subsection{CCPs discretization}

Figure 1 shows the 3D model of a tool cutting the surface of a workpiece in STS turning. The coordinate system on the upper left corner in Figure 5 represents lathe coordinate system. The processing of complex curved surfaces using STS turning refers to the relative movement of the cutting edge of the diamond tool and the surface of the workpiece, so as to achieve the removal of excess material on the surface of the workpiece. The CCPs are the position points on the workpiece's surface, which get into contact with the diamond tool, point P, as shown in Figure 1. Discretization must be performed to obtain CCPs.

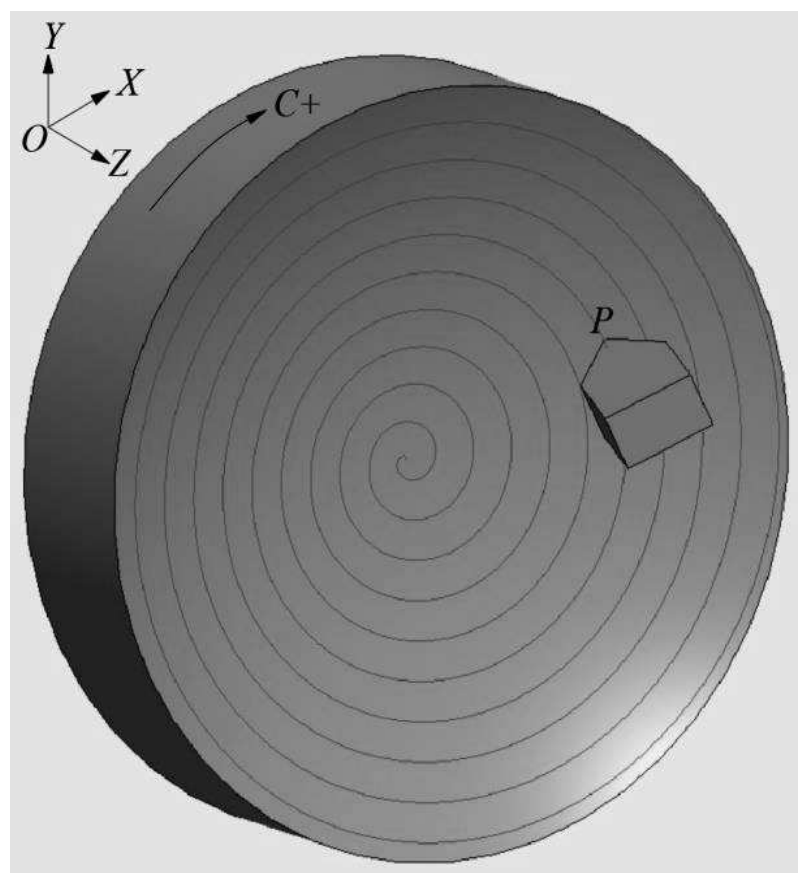

Fig. $13 D$ model of tool cutting workpiece's surface in STS turning

\section{Equal parameter discretization}

At present, equal parameter discretization method is mostly adopted to discretize CCPs, and this method mainly includes equal angle discretization method and equal arc length discretization method [9-10]. The equal angle discretization method controls the generation of CCPs through the discrete angle (the angle between two adjacent CCPs connected to the center point in the plane XOY) $\Delta \theta$. Its advantages are simplicity in calculation and ease of evaluation. However, the trajectory points that are closer to the central region are too dense, while the points of the outer trajectory are relatively sparse. As such, when the diameter of workpiece is larger, the discrete error will be larger in the outer of the workpiece. The equal arc length discretization method controls the generation of CCPs through the discrete arc length (the arc length between two adjacent CCPs in the plane XOY) $\Delta l$. In equal arc length discretization method, the CCPs near the center of the workpiece become very sparse and the machining trajectory's curvature in the center of the workpiece becomes larger, which causes the increase in the discrete error. In addition, Chen Xu et al. [9-10] proposed the comprehensive discrete method, but the type of the complex surface was not taken into consideration in these three methods. Using the same discretization methods for different types of complex surfaces' CCPs generation is unsuitable, and the discrete error is unpredictable and difficult to control.

\section{Equal height discretization}

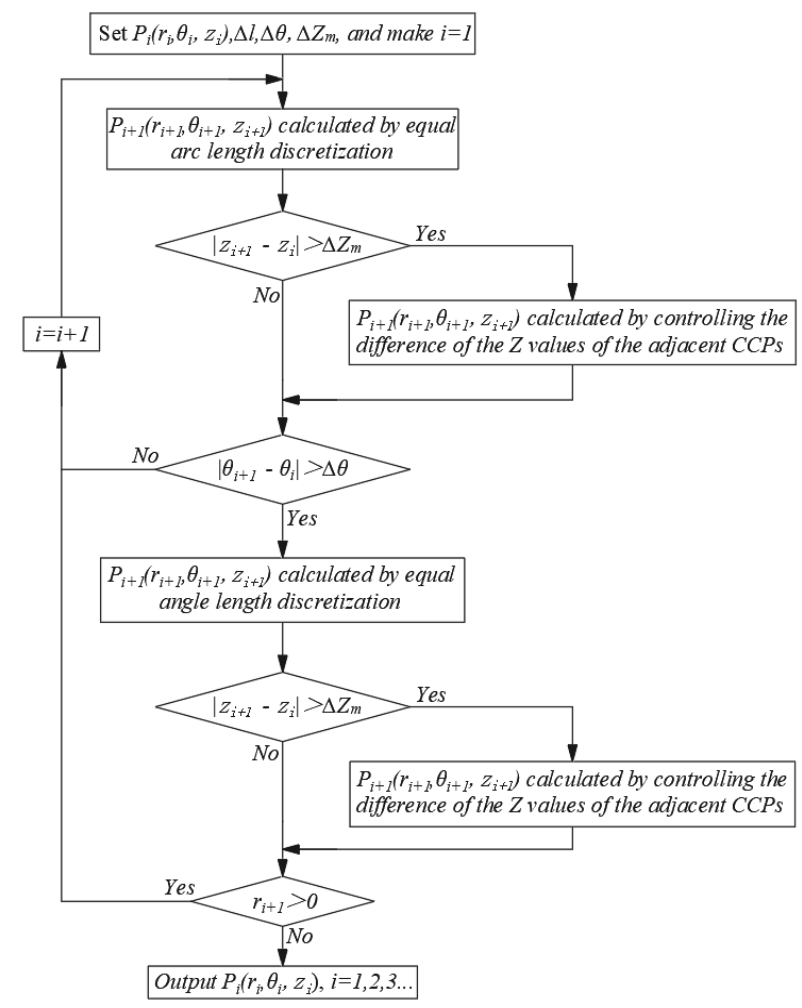

Fig. 2 Schematic diagram of the equal height discretization method

In order to reduce the discrete error and improve the machining accuracy, equal height discretization method is proposed in this paper. Equal height discretization method not only integrates the advantages of equal parameter discretization methods, but also takes the types of complex surfaces into consideration. It generates CCPs by controlling the height difference of 
the two adjacent CCPs. Therefore, the height difference of every two adjacent CCPs will be limited to a fixed range. This method does not control the generation of CCPs by controlling a single discrete angle or discrete arc length. Instead, it generates all CCPs by controlling the height difference between the CCP that has been sought and the next CCP to be sought. The height difference between every two adjacent CCPs is limited to a fixed range by using this method.

Figure 2 shows a schematic diagram of the equal height discretization method for the generation of CCPs. The "entry point" is $P_{1}$ in the outermost circle of the workpiece. Where, $P_{i}\left(r_{i}, \theta_{i}, z_{i}\right)$ represents the coordinate value of the CCPs in the cylindrical coordinate system, $r_{i}$ is the distance from the CCP $P_{i}$ to the workpiece center $\mathrm{O}, \theta_{i}$ is the angle from the CСP $P_{1}$ to the CСP $P_{i}$, and $z_{i}$ is the vector height of the CCP $P_{i} . \Delta l$ and $\Delta \theta$ are the same discrete arc length and discrete angle as defined above, which are called allowable discrete arc length and allowable discrete angle respectively here. $\Delta Z_{m}$ is the allowable discrete height difference between two adjacent CCPs. First, the coordinate value of the CCP $P_{i+1}$ is calculated using the equal arc length discretization method. If $\left|z_{i+1}-z_{i}\right|>\Delta Z_{m}$, the coordinate value of the CCP $P_{i+1}$ is calculated by controlling the difference between the $Z$ values of two adjacent CCPs. If $\left|z_{i+1}-z_{i}\right| \leq \Delta Z_{m}$, then judge whether $\mid \theta_{i+1}-$ $\theta_{i} \mid$ is greater than $\Delta \theta$. If $\left|\theta_{i+1}-\theta_{i}\right| \leq \Delta \theta$, then the next CCP is calculated. If $\left|\theta_{i+1}-\theta_{i}\right|>\Delta \theta$, the coordinate value of the CCP $P_{i+1}$ is calculated using the equal angle discretization method. If $\left|z_{i+1}-z_{i}\right|$ $>\Delta Z_{m}$, the coordinate value of the CCP $P_{i+1}$ is calculated by controlling the difference between the $Z$ values of two adjacent CCPs. Finally, it is judged whether $r_{i+1}$ is less than 0 . If $r_{i+1}<0$, the cycle algorithm ends, and all the coordinate values of the CCPs calculated are outputted.

The equal height discretization method calculates the coordinate value of the CCP $P_{i+1}$ by controlling the difference between the $\mathrm{Z}$ values of the adjacent CCPs by halving the angle of the adjacent CCPs. Figure 3 illustrates the schematic diagram of the method halving the angle of adjacent CCPs. Where, $P_{i}\left(r_{i}, \theta_{i}, z_{i}\right)$ is the coordinate value of the CCP that has been calculated, and $P_{i+1}\left(r_{i+1}, \theta_{i+1}, z_{i+1}\right)$ is the coordinate value of the CCP calculated by the equal parameter discrete method. Here, $\left|z_{i+1}-z_{i}\right|>\Delta Z_{m}$, the value $z_{i+1}$ needs to be controlled. First, the angle $\theta_{i}$ of the CСP $P_{i}$ and the angle $\theta_{i+1}$ of the CCP $P_{i+1}$ are halved and then re-assigned to $\theta_{i+1}$. Then, the coordinate value of the new CCP $P_{i+1}$ is calculated using formula (1). Finally, it is judged whether $\left|z_{i+1}-z_{i}\right|$ is greater than $\Delta Z_{m}$. If $\mid z_{i+1}-$ $z_{i} \mid>\Delta Z_{m}$, then the angle $\theta_{i}$ of the ССР $P_{i}$ and the angle $\theta_{i+1}$ of the CСP $P_{i+1}$ are halved and then re-assigned to $\theta_{i+1}$ again. $P_{i+1}\left(r_{i+1}, \theta_{i+1}, z_{i+1}\right)$ is outputted for the next step calculation of the equal height discrete method until the end of the loop algorithm.

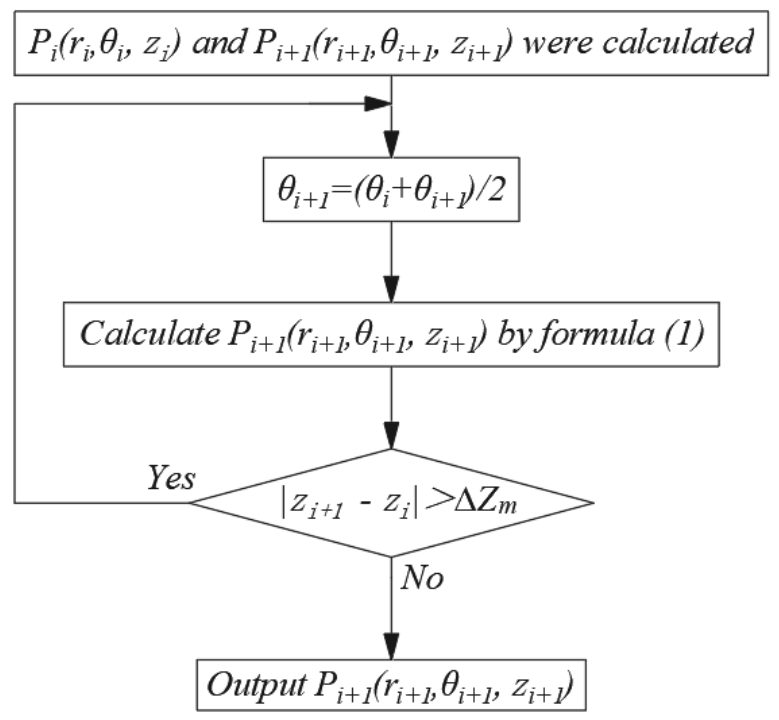

Fig. 3 The schematic diagram of the method halving the angle of adjacent CCPs

$$
\left\{\begin{array}{l}
r_{i}=R-\frac{a_{f}}{2 \pi} \theta_{i} \\
z_{i}=f\left(r_{i}, \theta_{i}\right)
\end{array} \quad(i=1,2,3 \ldots)\right.
$$

Where, $\left(r_{i}, \theta_{i}, z_{i}\right)$ is the coordinate value of the CCP $P_{i}, R$ is the radius of the workpiece, and $a_{f}$ is the feed per revolution of the $\mathrm{X}$ axis.

The equal height discretization method is essentially a combination of the above-mentioned methods. If the $\Delta Z_{m}$ value is larger, then the method is a comprehensive discretization method composed of equal angle discretization and equal arc discretization method. If the $\Delta Z_{m}$ value is smaller, then the method will not use equal angle discretization and equal arc discretization method, but obtains the coordinate values of the CCPs by controlling the difference between the $\mathrm{Z}$ values of two adjacent CCPs.

Through the equal height discretization method, most of the CCPs in the outer ring of the workpiece were obtained by the equal arc length discretization method, and most of the CCPs in the inner ring were obtained by the equal angle discretization method. The remaining CCPs at the positions where the curvature is larger on the surface were obtained by controlling the difference between the $Z$ values of two adjacent CCPs. Therefore, this method retains the advantages of the two equal parameter discretization methods, and takes into account the surface shape of complex curved surfaces, and can control the discrete error within a small range.

As shown in Figure 4(a) and 4(b), the CCPs of a 
sinusoidal array surface were discretized by equal height discretization method. Equal height discre-

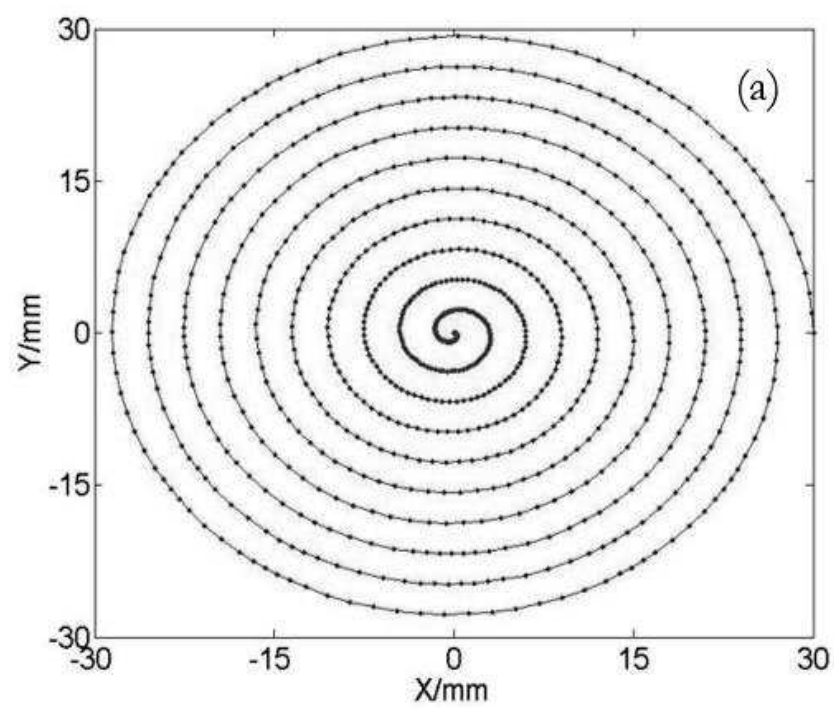

tization limits the height increment between two adjacent CCPs where the curvature of the surface was large, thus the discrete error was reduced.

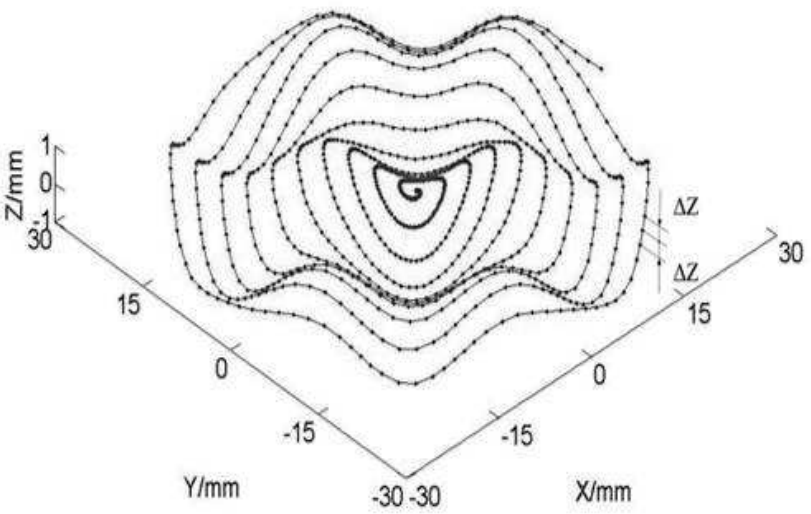

Fig. 4 Schematic diagram of equal height discretization for sinusoidal array surface (a) Schematic diagram of XY plane, (b) Schematic diagram of $3 D$ space

\subsection{Cutting tools geometry compensation}

In the actual cutting process, the contact point between the tool nose and the workpiece is not a fixed point on the tool nose, but may be a series of constantly changing points on the arc of tool nose. Therefore, it is necessary to specify a fixed point on the tool to determine the position of the tool during the cutting process. This point is called the cutting location point (CLP). Owing to the complexity of the complex surface and the use of diamond turning tool in the experiment with an arc of tool nose, the contact point between the tool nose and the machined surface is inconsistent at different positions on the surface. The movement of the tool cannot be controlled simply by the coordinates of the CCPs. The discrete CCPs must be converted into discrete CLPs through tool geometry compensation. In this paper, the rake angle of the tool is $0^{\circ}$, back angle is $15^{\circ}$, and the arc radius of tool nose is $0.5 \mathrm{~mm}$, so the tool geometry compensation is tool radius compensation. The tool shape compensation algorithm used in this paper is the Zdirection compensation algorithm proposed by Wang [9] and Chen et al. [16]. This algorithm can ensure the dynamic performance of the $\mathrm{X}$ axis and improve the machining accuracy.

\section{Trajectory interpolation of cutting loca- tion points}

\subsection{Processing of interpolation nodes}

The processing trajectory interpolation is required after obtaining the discrete CLPs. The discrete CLPs were the interpolation nodes. In this experimental platform, the integrated multi-axis controller 400 (IMAC400) can be used to complete the processing path interpolation. The PVT (Position-velocity-time) interpolation mode supported by IMAC is used for the interpolation of the machining trajectory [9]. The parameters required for this mode are the position of the CLP, the velocity at the CLP, and the time interval between two adjacent points. The mathematical essence of PVT mode is segment cubic Hermite polynomial interpolation [19]. This mode can generate a unique three order curve according to the given parameters, so it can realize precise control of the motion.

Uniform node interpolation means that the interpolation nodes are arranged uniformly in the $\mathrm{X}$ direction, while non-uniform node interpolation means that the interpolation nodes are arranged unevenly in the $\mathrm{X}$ direction. Since the curvatures of different positions on the tool path curve are different, if the same uniform node interpolations are used at different positions, the interpolation error will be larger at the position with larger curvature.

In order to reduce the interpolation error, a new interpolation algorithm referred to as non-uniform node was put forward in this paper. This method achieves the purpose of non-uniformity processing by increasing the interpolation nodes at the position where the curvature of the trajectory curve is larger and reducing the interpolation nodes at the position where the curvature of the trajectory curve is smaller, thereby reducing the interpolation error. The interpolation error is larger where the curvature of the curve is larger because of the shape preserving characteristics of the Hermite interpolation curve [16]. However, the interpolation nodes were generally uniform, so the 
interpolation error of PVT was influenced by the curvature of the curve, whereby the larger the curvature, the larger the interpolation error [20]. Therefore, selection of more interpolation nodes in the large curvature of the curve is an effective method for reducing interpolation error. Figure 5 shows the comparison result of using uniform nodes and non-uniform nodes to interpolate the sinusoidal curve. Figure 5(a) shows the uniform nodes interpolation and the non-uniform

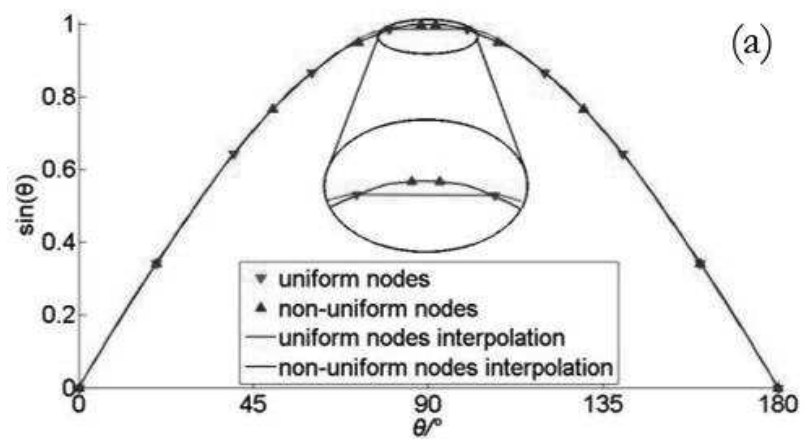

nodes interpolation of the sinusoidal curve. It can be seen that non-uniform nodes interpolation curve with ten interpolation nodes was closer to the true curve. Figure 5(b) shows the interpolation error of the uniform nodes interpolation and the non-uniform nodes interpolation of the sinusoidal curve. It can be seen that non-uniform nodes interpolation can control the error distribution and reduce the maximum interpolation error by about $70 \%$.

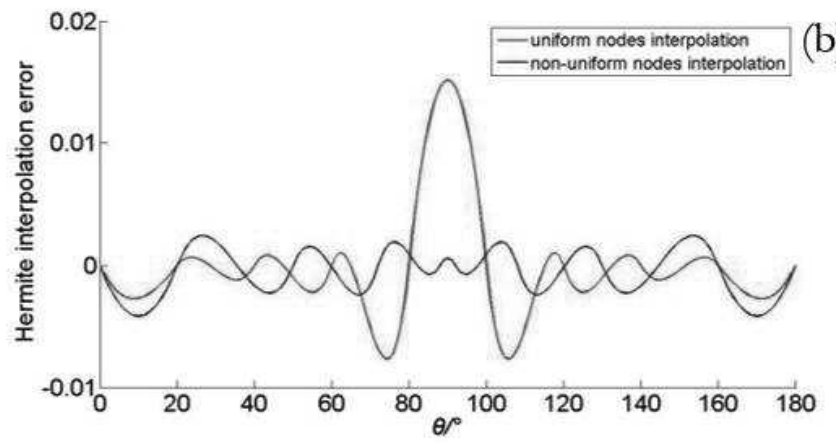

Fig. 5 Comparison of uniform nodes interpolation and non-uniform nodes interpolation (a) Comparison of sinusoidal interpolation curve, (b) Comparison of sinusoidal curve interpolation error

In addition, the interpolation error can be further reduced by densifying the interpolation nodes. Figure 6 shows the method of densification of interpolation nodes.

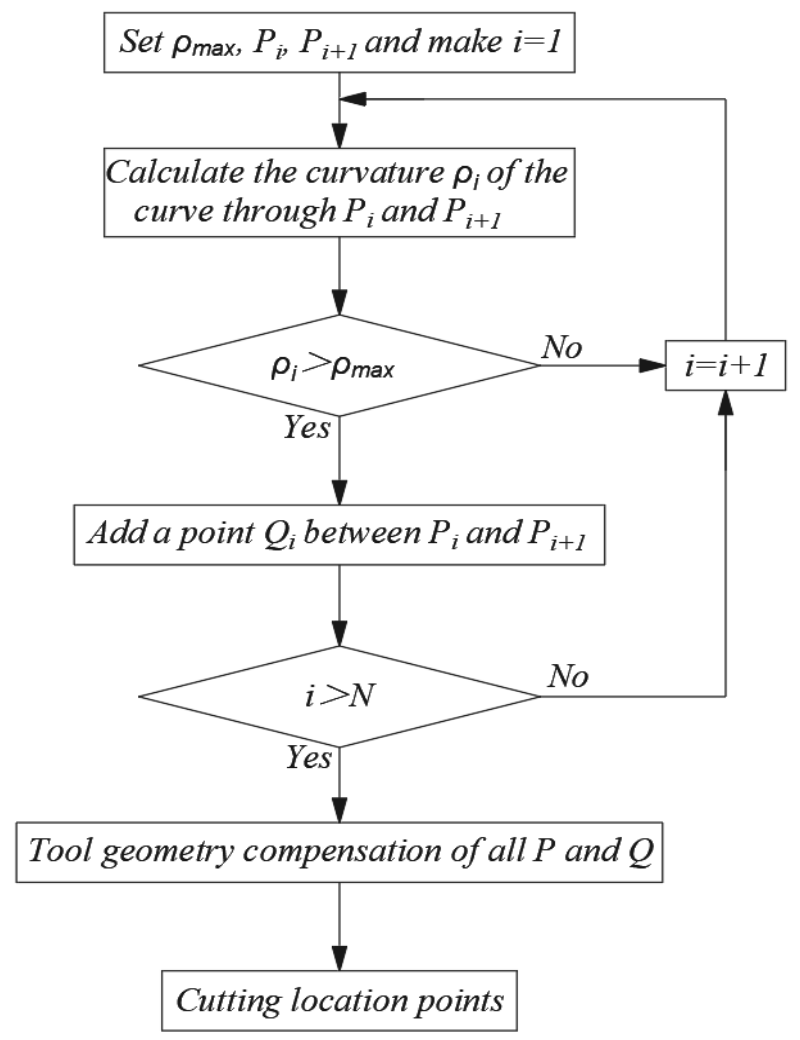

Fig. 6 The method of densification of interpolation nodes

Where $\rho_{\max }$ is the maximum curvature to judge whether the nodes start to increase. $P_{i}$ and $P_{i+1}$ are the CCPs discretized by equal height discretization method, and $\mathrm{N}$ is the total number of CCPs.

\subsection{Calculation of interpolation nodes velocity}

The key step of the PVT interpolation mode is to calculate the velocity of the CLPs, which mainly consists of velocity calculation of the interpolation nodes. Segment cubic Hermite polynomial interpolation can guarantee that the velocity of each interpolation node is continuous, but it cannot guarantee that the acceleration of each interpolation node is continuous. Segment cubic spline interpolation, however, can guarantee the velocity and acceleration are both continuous, simultaneously [9]. Figure 7 shows the difference in the interpolation of sinusoidal curve using segment cubic Hermite interpolation and segment cubic spline interpolation. It can be seen that the segment cubic spline interpolation curve is smoother with the same interpolation nodes. At the position of large curvature, the interpolation error of the segment cubic Hermite interpolation is larger than that of the segment cubic spline interpolation.

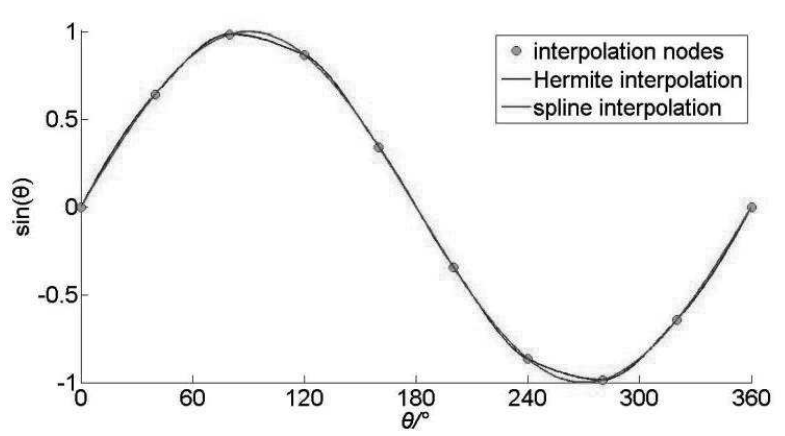

Fig. 7 Comparison of the interpolation results of two interpolation methods on sinusoidal curve 
The difference between the segment cubic spline interpolation and the segment cubic Hermite interpolation lies in the velocity calculation of the interpola-

$$
I^{(k)}(x)=f_{k} \alpha_{k}(x)+f_{k+1} \alpha_{k+1}(x)+f_{k}{ }^{\prime} \beta_{k}
$$

Where, $I^{(k)}(x)$ is the cubic interpolation polynomial on the interval $\left[x_{k}, x_{k+1}\right], f_{k}$ and $f_{k}{ }^{\prime}$ are the function value and the first derivative value of the interpolation nodes, respectively, and $x_{0}, x_{1}, \cdots, x_{n}$ are the interpolation nodes. $\alpha_{k}(x)$ and $\beta_{k}(x)$ are the interpolation basis functions, which can be expressed as shown in Equation (3):

$$
\begin{aligned}
& \left\{\begin{array}{l}
\alpha_{i}(x)=\left(\frac{x-x_{j}}{x_{i}-x_{j}}\right)^{2}\left(1+2 \frac{x-x_{i}}{x_{j}-x_{i}}\right) \\
\beta_{i}(x)=\left(\frac{x-x_{j}}{x_{i}-x_{j}}\right)^{2}\left(x-x_{i}\right)
\end{array}\right. \\
& I\left(x_{k}\right)=f_{k}, I^{\prime}\left(x_{k}\right)=f_{k}{ }^{\prime}, I^{\prime \prime}\left(x_{k}\right)=f_{k}{ }^{\prime \prime}, k=0,1, \ldots, n-1
\end{aligned}
$$

Therefore, the condition expressed in Equation (5) can guarantee that the acceleration of each interpolation node is continuous, and the corresponding interpolation functions can be obtained by the method of undetermined coefficients with $I^{\prime}\left(x_{k}\right)=m_{k}$ or $I^{\prime \prime}\left(x_{k}\right)=M_{k}$.

When the first order coefficient $I^{\prime}\left(x_{k}\right)=m_{k}$ is an undetermined coefficient, $I(x), I^{\prime}(x)$ and $I^{\prime \prime}(x)$ can

$$
\begin{gathered}
I^{\prime \prime}(x)=\left[\frac{6}{h_{k}^{2}}-\frac{12}{h_{k}^{3}}\left(x_{k+1}-x\right)\right] f_{k}+\left[\frac{6}{h_{k}^{2}}-\frac{12}{h_{k}^{3}}\left(x-x_{k}\right)\right] f_{k+1}+\left[\frac{2}{h_{k}}-\frac{6}{h_{k}^{2}}\left(x_{k+1}-x\right)\right] m_{k}-\left[\frac{2}{h_{k}}-\frac{6}{h_{k}^{2}}\left(x-x_{k}\right)\right] m_{k+1} \\
\frac{h_{k}}{h_{k}+h_{k+1}} m_{k-1}+2 m_{k}+\frac{h_{k+1}}{h_{k}+h_{k+1}} m_{k+1}=3\left(\frac{h_{k}}{h_{k}+h_{k+1}} \frac{f_{k}-f_{k-1}}{h_{k-1}}+\frac{h_{k+1}}{h_{k}+h_{k+1}} \frac{f_{k+1}-f_{k}}{h_{k}}\right)
\end{gathered}
$$

When the second order coefficient $I^{\prime \prime}\left(x_{k}\right)=$ $M_{k}$ is an undetermined coefficient, $I(x)$ and $I^{\prime}(x)$ can be calculated by combining condition (5) and Equation (2). Equation (8) represents the velocity formula. Combined with the condition of derivatives

$$
\begin{gathered}
I^{\prime}(x)=-M_{k} \frac{\left(x_{k+1}-x\right)^{2}}{2 h_{k}}+M_{k+1} \frac{\left(x-x_{k}\right)^{2}}{2 h_{k}}+\frac{f_{k+1}-f_{k}}{h_{k}}-\frac{M_{k+1}-M_{k}}{6} h_{k} \\
\frac{h_{k-1}}{h_{k-1}+h_{k}} M_{k-1}+2 M_{k}+\frac{h_{k}}{h_{k-1}+h_{k}} M_{k+1}=6 f\left[x_{k-1}, x_{k}, x_{k+1}\right]
\end{gathered}
$$

Equations (7) and (9) are both undetermined equations, so the undetermined coefficients should be calculated through appropriate boundary conditions (such as natural boundary conditions) [22]. The segment cubic spline interpolation polynomials and the velocity of interpolation nodes are then obtained. The second order continuity of the interpolation nodes can be ensured by transforming the segment cubic Hermite polynomial interpolation into segment cubic spline interpolation using Equations (10). The interpolation error can be further reduced by non-uniformization of the interpolation nodes. Figure 8 shows the interpolation results and the interpolation error distribution of sinusoidal curve by using segment cubic Hermite interpolation and segment cubic spline interpolation with the same non-uniform interpolation nodes. It can be seen that the interpolation curves of the two methods are approximately the same from Figure 8 (a). It can also be seen that the interpolation error decreased by about $90 \%$ by transforming segment cubic Hermite interpolation into segment cubic spline interpolation in Figure 8(b). 

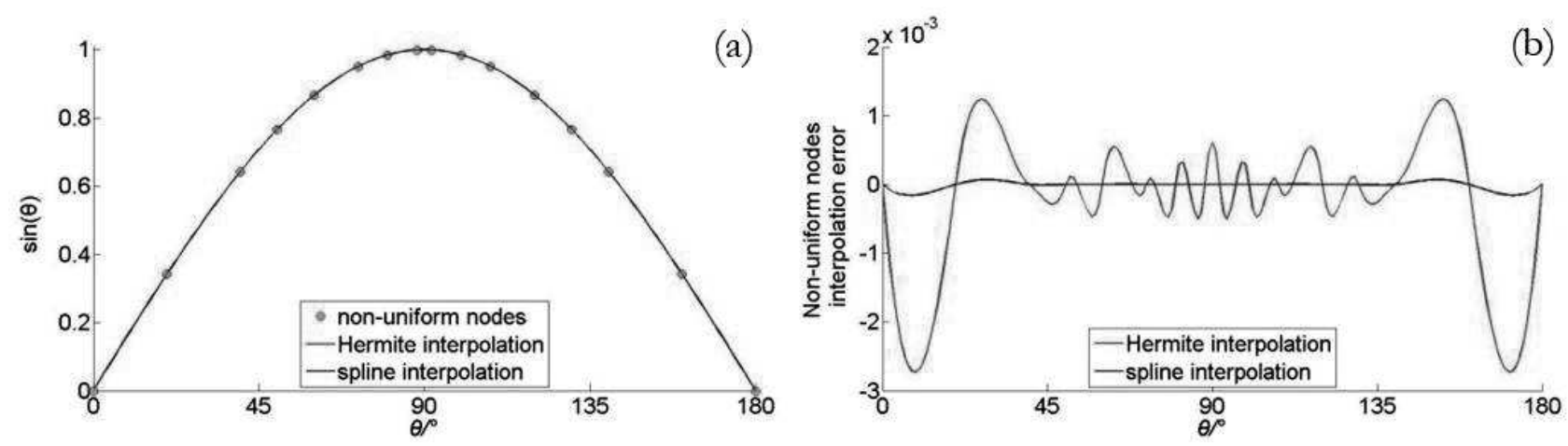

Fig. 8 Comparison of two interpolation methods under non-uniformity processing (a) Comparison of sinusoidal interpolation curve, (b) Comparison of interpolation error of sinusoidal curve

\section{Simulation analysis}

After discretization of CCPs and trajectory interpolation of the CLPs, the STS turning of the complex surface can be realized. The toric surface and sinusoidal array surface were chosen as the simulation analysis case for the verification of tool path generation.

\subsection{Toric surface}

The toric surface is a non-rotationally symmetrical surface. Two different diopters are formed due to its different steepness in two mutually perpendicular directions. Based on this feature, it is widely used in the correction of eye astigmatism. Its mathematical expression is shown in Equation (14) [23]:

$$
z=R+a-\sqrt{\left(R+\sqrt{a^{2}-y^{2}}\right)^{2}-x^{2}}
$$

The related parameters of this simulation experiment are described in the table below.

Tab. 1 Simulation parameters of tool path planning of the toric surface

\begin{tabular}{lclc}
\hline \multicolumn{1}{c}{ Name of parameters } & $\begin{array}{c}\text { Parameter's } \\
\text { value }\end{array}$ & \multicolumn{1}{c}{ Name of parameters } & $\begin{array}{c}\text { Parameter's } \\
\text { value }\end{array}$ \\
\hline Radius of orthogonal arc $R(\mathrm{~mm})$ & 140 & Radius of workpiece $R(\mathrm{~mm})$ & 20 \\
Radius of basic arc $a(\mathrm{~mm})$ & 100 & Discretization height $\Delta Z(\mathrm{~mm})$ & 0.04 \\
Feed velocity $a_{f}(\mathrm{~mm} / \mathrm{r})$ & 0.5 & Discretization arc length $\Delta l(\mathrm{~mm})$ & 1 \\
Tool radius $R_{t}(\mathrm{~mm})$ & 0.2 & Discretization angle $\Delta \theta\left(^{\circ}\right)$ & 5 \\
\hline
\end{tabular}

Figure 9 shows the designed surface of the toric surface and the distribution of the CCPs obtained by equal height discretization method.

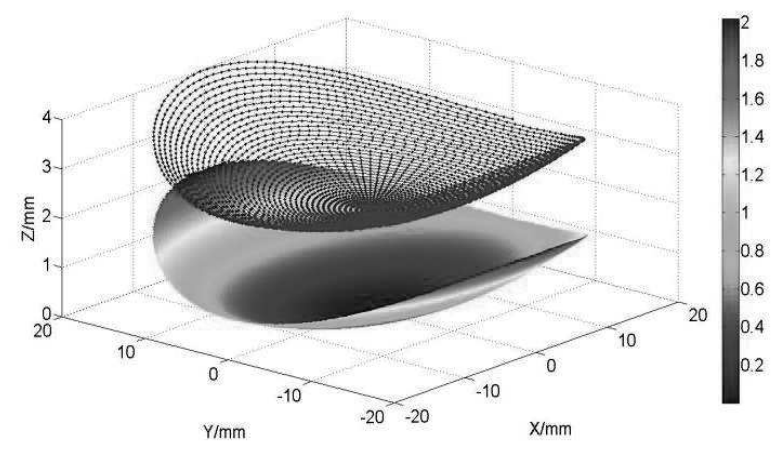

Fig. 9 The designed surface of the toric surface and the distribution of the CCPs obtained by equal height discretization method

The CCPs obtained by the three discretization methods described above were interpolated by the segment cubic spline interpolation method. The difference of the $Z$ values between the interpolation point and the theoretical point is called the discrete error.
Figure 10 shows the distribution of the height difference $\Delta \mathrm{Z}$ of every two adjacent CCPs obtained by the three discretization methods above, and the corresponding discrete errors of the three discretization methods.

It can be seen that the distribution of CCPs discretized by both equal angle and equal arc length discretization method were non-uniform in $\mathrm{Z}$ direction from Figure 10(a) and 10(c). For equal angle discretization method, the difference of the $\mathrm{Z}$ values $(\Delta \mathrm{Z})$ of the adjacent CCPs of the outer ring was larger since the CCPs of the outer ring were sparse relative to the inner ring, which lead to a larger discrete error in the outer ring, as shown in Figure 10(b). For equal arc length discretization method, the difference of the $Z$ values $(\Delta Z)$ of the adjacent CCPs of the inner ring was larger since the CCPs of the inner ring were relatively sparse, which lead to a larger discrete error in the inner ring, as shown in Figure 10(d).

From Figure 10(e) and 10(f), it is evident that for equal height discretization method, the distribution of CCPs was uniform in $Z$ direction and the values of $\Delta Z$ were limited to a fixed small range and consequently, the discrete error was reduced. Since the distribution 
of CCPs in the $Z$ direction was relatively uniform, the velocity and acceleration of the $Z$ axis varied within a small range. This in turn reduced the vibration of the machining process and improved the machining accuracy. It can be seen that the order of the maximum discretization error of the three discretization met-
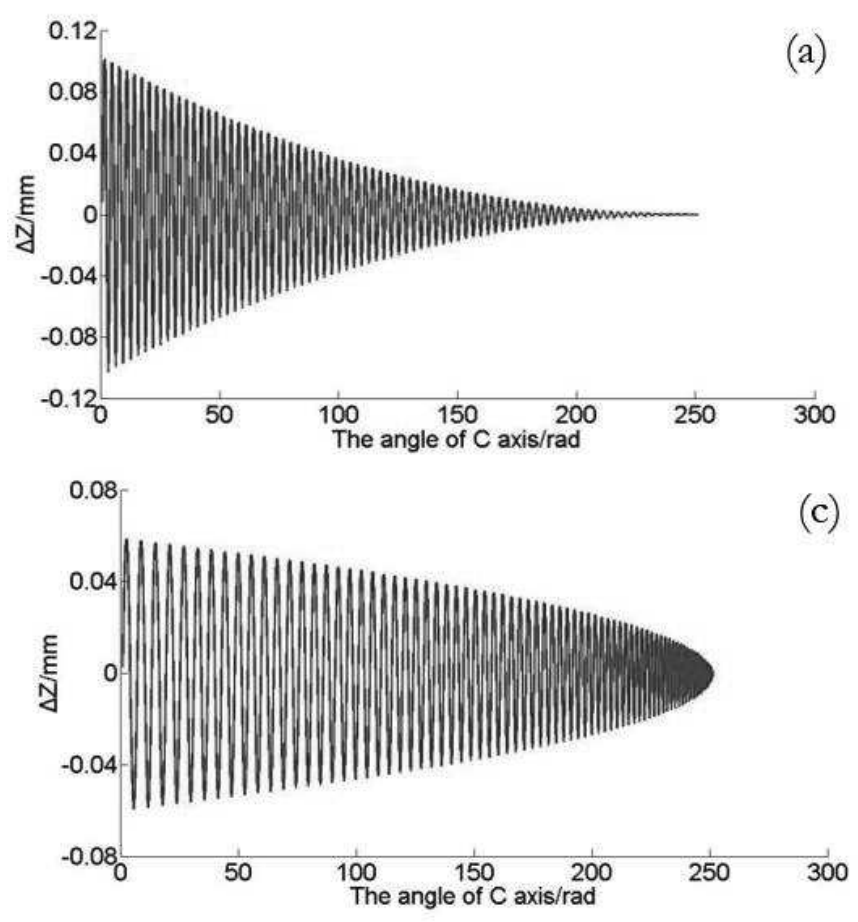

(c)

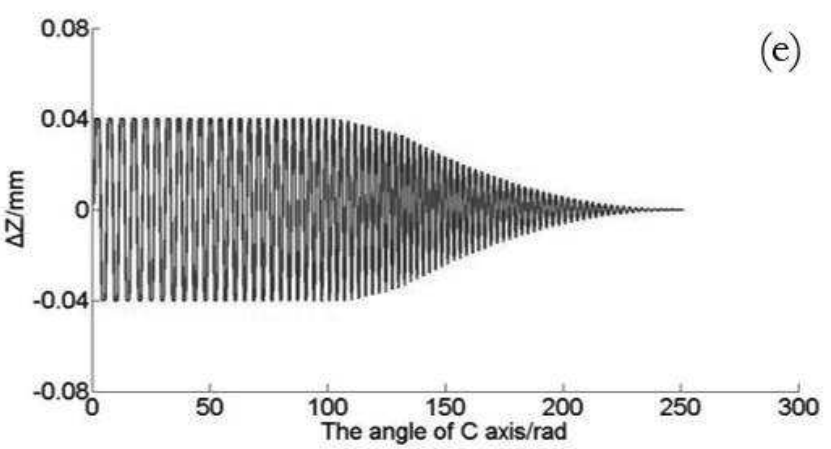

hods is: equal height discretization method <equal angle discretization method<equal arc length discretization method from Figure 10(b), (d) and (f). Compared with the equal angle discretization method, the equal height discretization method can reduce the discretization error by about $70 \%$.
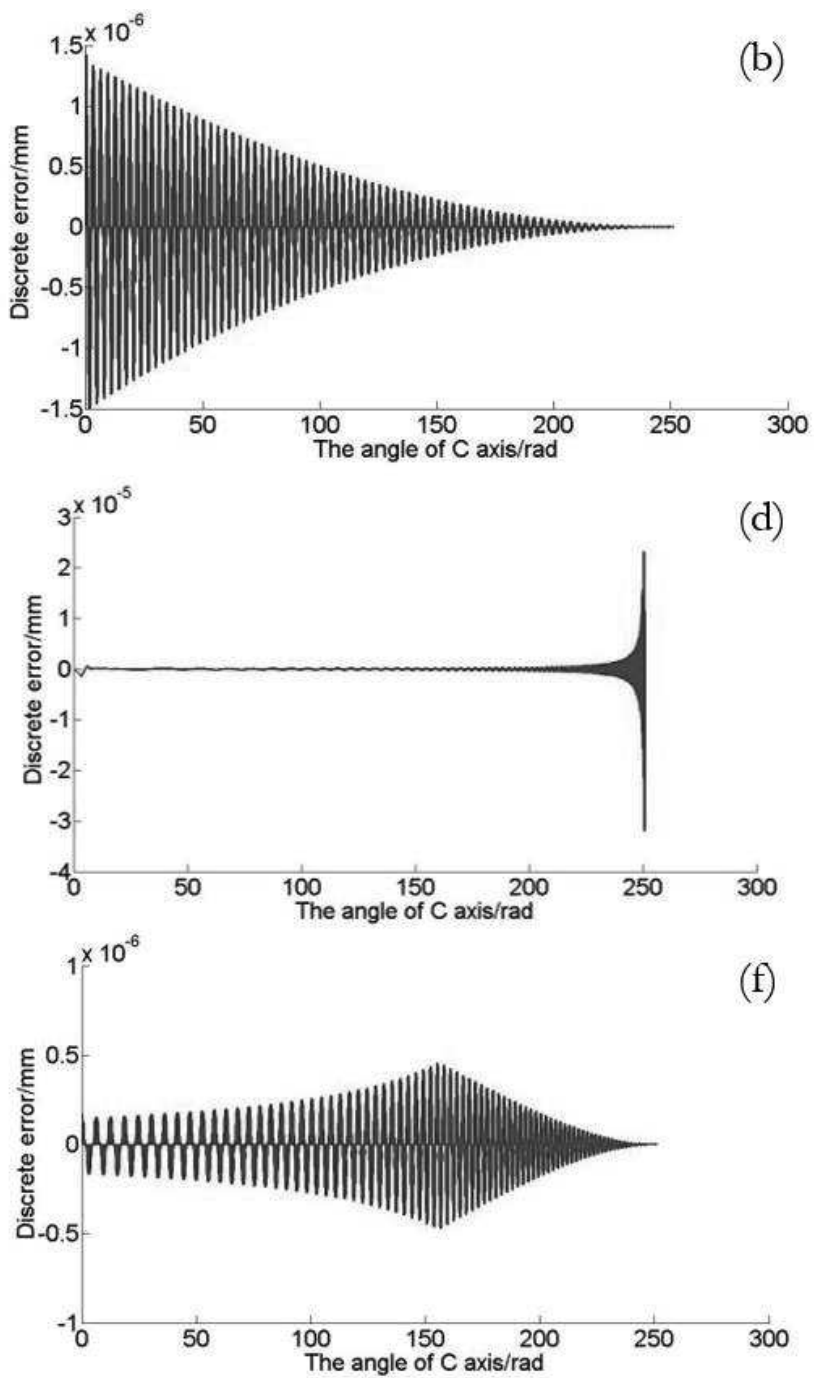

Fig. 10 The distribution of $\Delta Z$ of every two adjacent CCPs obtained by the three discretization methods and the corresponding discrete error, (a) The distribution of $\Delta Z$ by equal angle discretization, (b) The discrete error of equal angle discretization, (c) The distribution of $\Delta Z$ by equal arc length discretization, (d) The discrete error of equal arc length discretization, (e) The distribution of $\Delta Z$ by equal height discretization, (f) The discrete error of equal height discretization

Segment cubic Hermite interpolation was used to interpolate the CLPs after the tool geometry compensation for the analysis of PVT interpolation error. The difference of the $Z$ values between the interpolation points and the theoretical points was called the interpolation error. Figures 11(a) and 11(b) show the PVT interpolation error distributions obtained by using uniform nodes segment cubic Hermite interpolation and non-uniform nodes segment cubic Hermite interpolation. It can be seen that the interpolation error was reduced by about $70 \%$ by using non-uniform node interpolation under the same conditions. Figure 12 represents the interpolation error of PVT by using the triangle rotary method to convert the segment cubic Hermite interpolation into the segment cubic spline interpolation. Under the natural boundary conditions, the velocity of interpolation points calculated by the triangle rotary method and the triangle bending moment method was basically the same, and so was the interpolation error. However, both were about two orders of magnitude smaller than the error of segment cubic Hermite interpolation. 

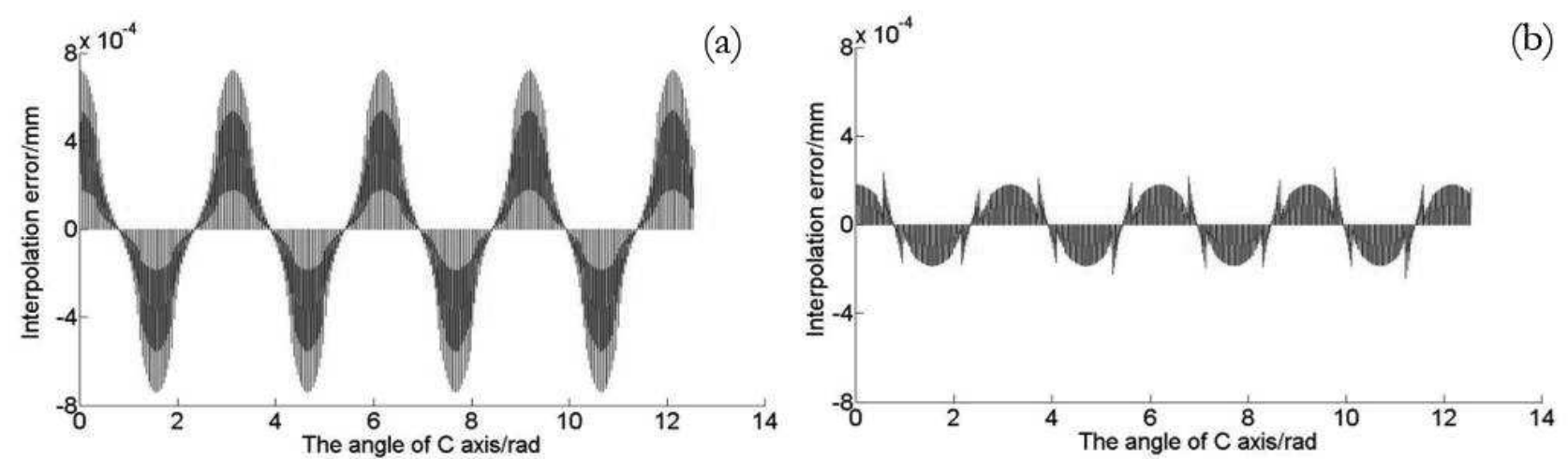

Fig. 11 The interpolation error of uniform nodes segment cubic Hermite interpolation and non-uniform nodes segment cubic, Hermite interpolation, (a) The interpolation error of uniform nodes segment cubic Hermite interpolation, (b) The interpolation error of non-uniform nodes segment cubic Hermite interpolation

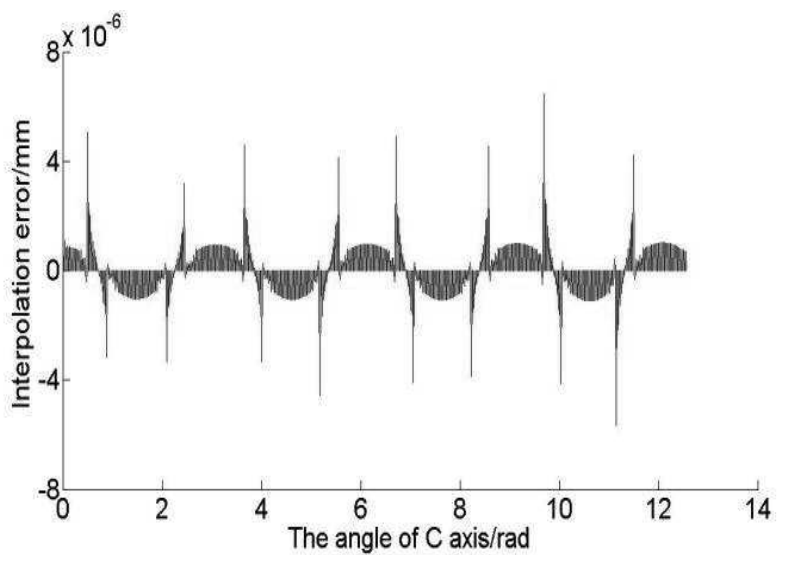

Fig. 12 The interpolation error of non-uniform nodes segment cubic spline interpolation

\subsection{Sinusoidal array surface}

The array surface means the repetition of the same or similar surface type, and is widely used in laser welding, electronic components and lighting systems [24]. The one-time molding process of the array surface can improve the installation accuracy and production efficiency, and avoid the shortcomings of the non-onetime molding process. The sinusoidal array surface is a common array surface, and its mathematical expression is given by Equation (11) [16]:

$$
z=A \sin \left(\frac{2 \pi x}{\omega}\right) \sin \left(\frac{2 \pi y}{\omega}\right)
$$

The related parameters of this simulation experiment are described in the table below.

Tab. 2 Simulation parameters of tool path planning of the sinusoidal array surface

\begin{tabular}{lclc}
\hline Name of parameters & Parameter's value & \multicolumn{1}{c}{ Name of parameters } & Parameter's value \\
\hline Amplitude $A(\mathrm{~mm})$ & 1 & Radius of workpiece $R(\mathrm{~mm})$ & 30 \\
Wave length $\omega(\mathrm{mm})$ & 30 & Discretization height $\Delta Z(\mathrm{~mm})$ & 0.1 \\
Feed velocity $a_{f}(\mathrm{~mm} / \mathrm{r})$ & 0.5 & Discretization arc length $\Delta l(\mathrm{~mm})$ & 1 \\
Tool radius $R_{t}(\mathrm{~mm})$ & 0.2 & Discretization angle $\Delta \theta\left(^{\circ}\right)$ & 3 \\
\hline
\end{tabular}

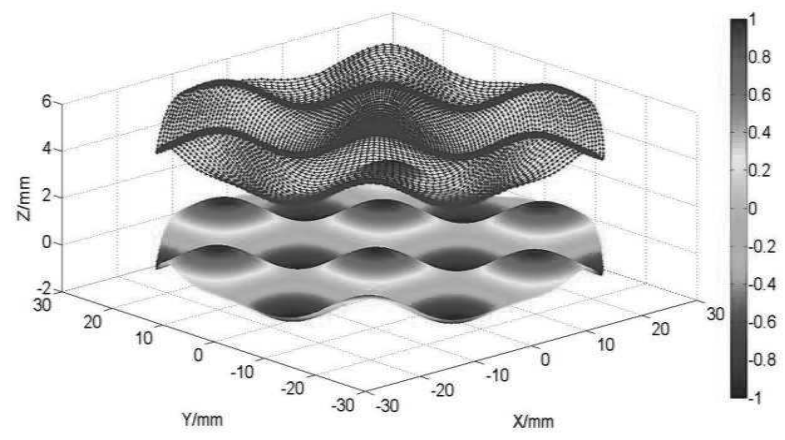

Fig. 13 The designed surface of the sinusoidal array surface and the distribution of the CCPs obtained by equal height discretization method
Figure 13 shows the designed surface of the sinusoidal array surface and the distribution of the CCPs obtained by equal height discretization method.

Figure 14 shows the distribution of the height difference $\Delta Z$ of every two adjacent CCPs obtained by the three discretization methods above, and the corresponding discrete errors of the three discretization methods. By comparison, it was found that the equal height discretization method reduced the discrete error and was superior to the other two discretization methods. This was the same case for the toric surface. Compared with the equal angle discretization method, the equal height discretization method can reduce the discretization error by about $80 \%$. 

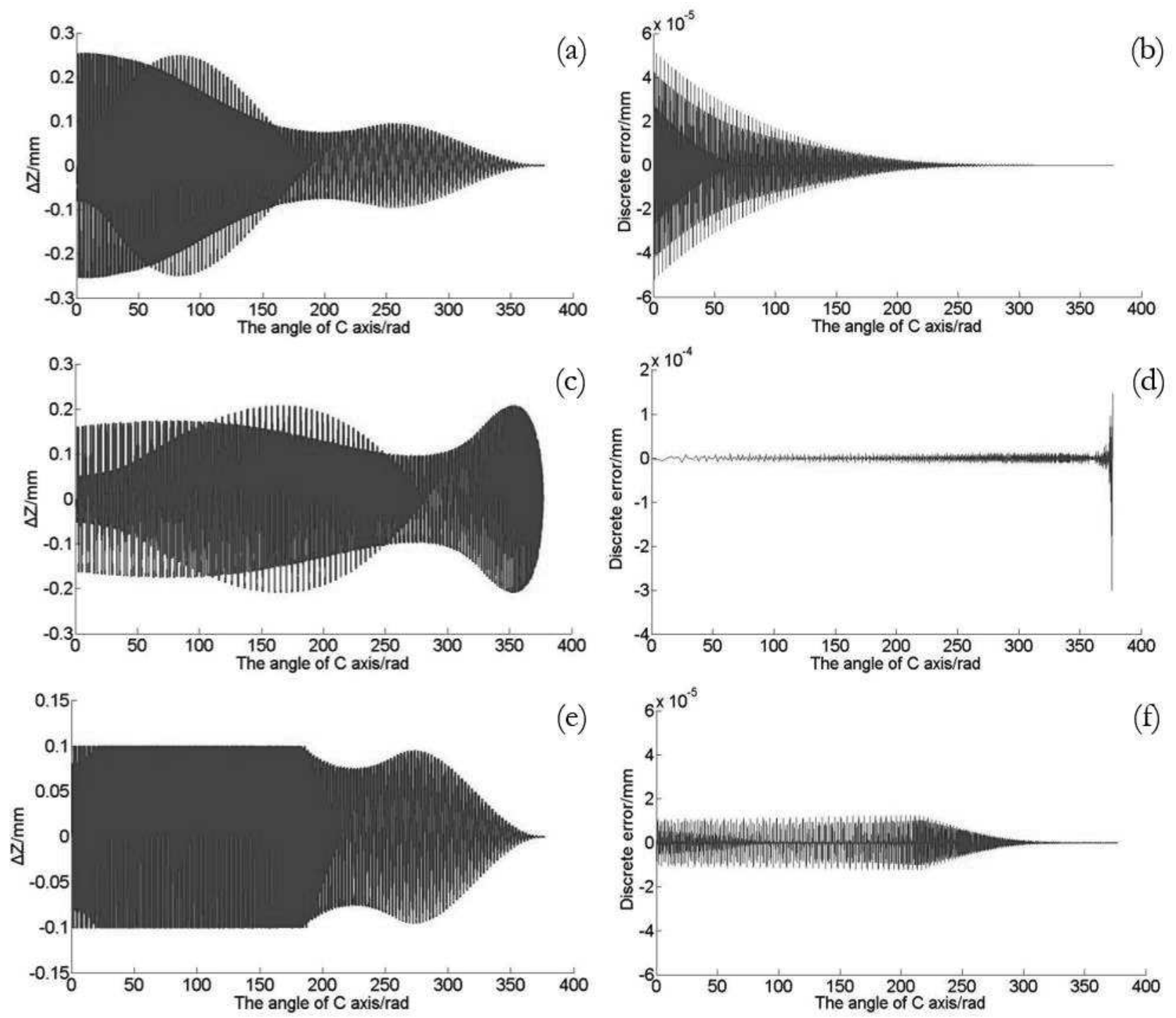

Fig. 14 The distribution of $\Delta Z$ of every two adjacent CCPs obtained by the three discretization methods and the corresponding discrete error, (a) The distribution of $\Delta Z$ by equal angle discretization, (b) The discrete error of equal angle discretization, (c) The distribution of $\Delta Z$ by equal arc length discretization, (d) The discrete error of equal arc length discretization, (e) The distribution of $\Delta Z$ by equal height discretization, (f) The discrete error of equal height discretization
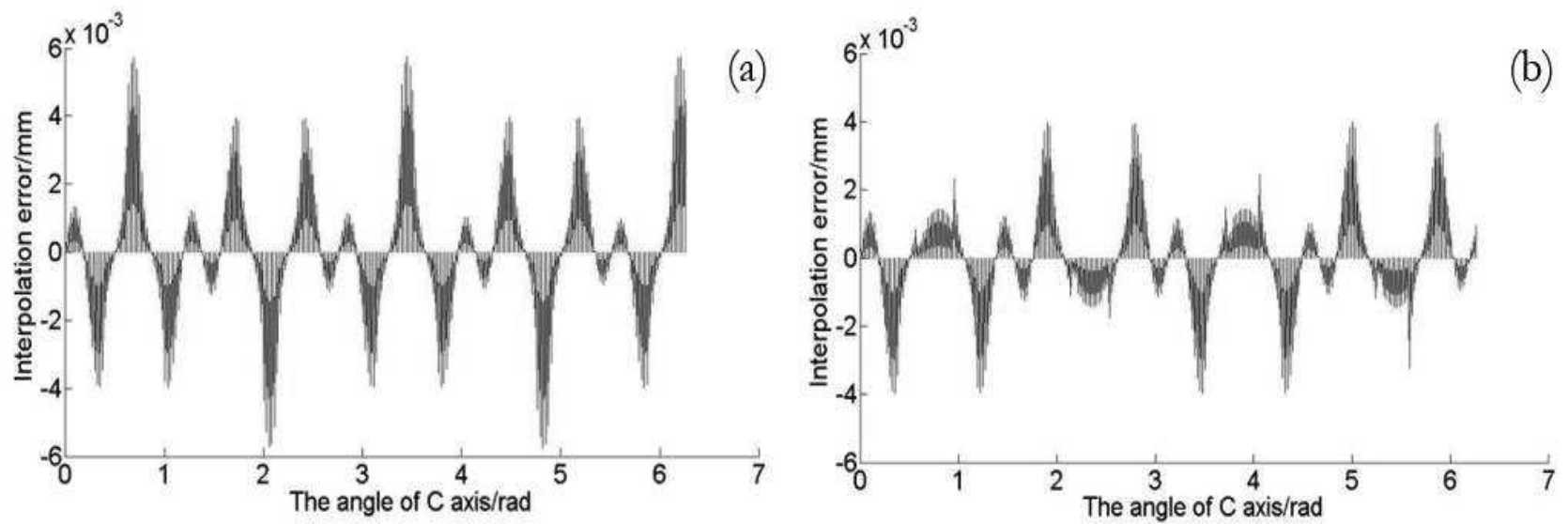

Fig. 15 The interpolation error of uniform nodes segment cubic Hermite interpolation and non-uniform nodes segment cubic, Hermite interpolation, (a) The interpolation error of uniform nodes segment cubic Hermite interpolation, (b) The interpolation error of non-uniform nodes segment cubic Hermite interpolation 
Figure 15 shows the PVT interpolation error distributions obtained by using uniform nodes segment cubic Hermite interpolation and non-uniform nodes segment cubic Hermite interpolation. It can be seen that the interpolation error was reduced by about $30 \%$ by using non-uniform nodes interpolation under the same conditions. Figure 16 represents the interpolation error of PVT by using the triangle rotary method to convert the segment cubic Hermite interpolation into the segment cubic spline interpolation. Similar to the toric surface, the maximum interpolation error was about two orders of magnitude smaller than the segment cubic Hermite interpolation error under the natural boundary conditions.

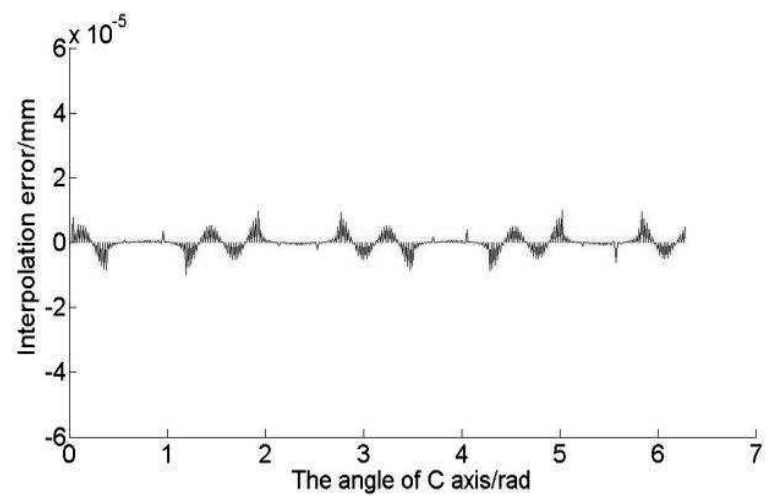

Fig. 16 The interpolation error of non-uniform nodes segment cubic spline interpolation

\section{Processing experiment}

According to the theoretical analysis and simulation analysis above, the simulation results were verified by experiments. Firstly, programs suitable for STS turning and can also automatically generate machining code (NC code) were written in MATLAB using the algorithm above. Then, the processing of the toric surface and the sinusoidal array surface was completed on the experimental device developed by our laboratory. Figure 17 shows the experimental platform of STS turning used in the experiment. The material of workpieces was PMMA (polymethyl methacrylate), the feed speed was $0.05 \mathrm{~mm} / \mathrm{r}$, and other processing parameters were the same as the simulation parameters.

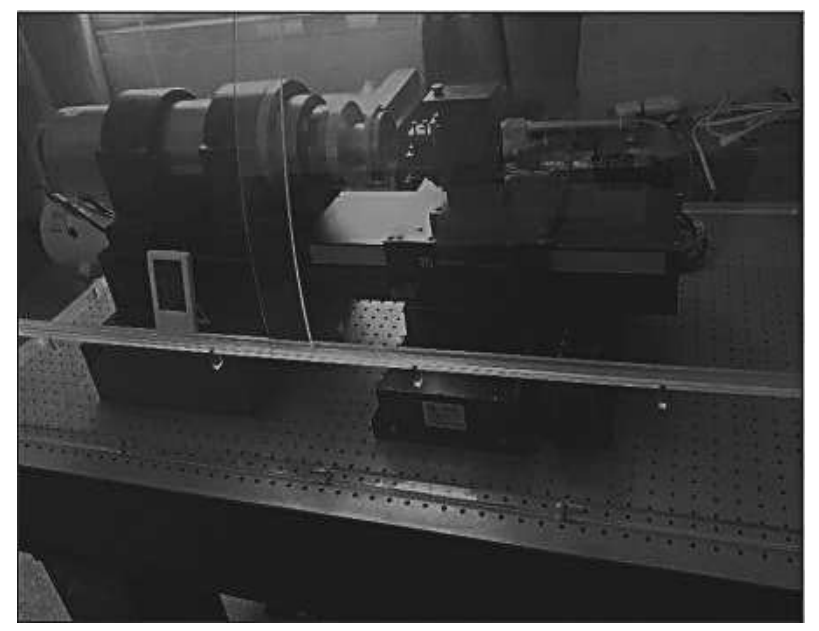

Fig. 17 The experimental platform of STS turning

Three discrete methods and uniform node segmentation cubic Hermite interpolation were used to complete the processing of toric surface. Figure 18 shows the machined workpieces of toric surface. In order to evaluate the surface smoothness of the machined workpieces, the surface roughness of the workpieces was measured by the JB-4C contact surface roughness measuring instrument. Due to the different discrete methods, the discrete errors were also different. According to the theoretical analysis above, the inner and outer rings of the workpiece were measured and compared. Figure 19 shows the measurement result of the surface roughness of the workpieces.

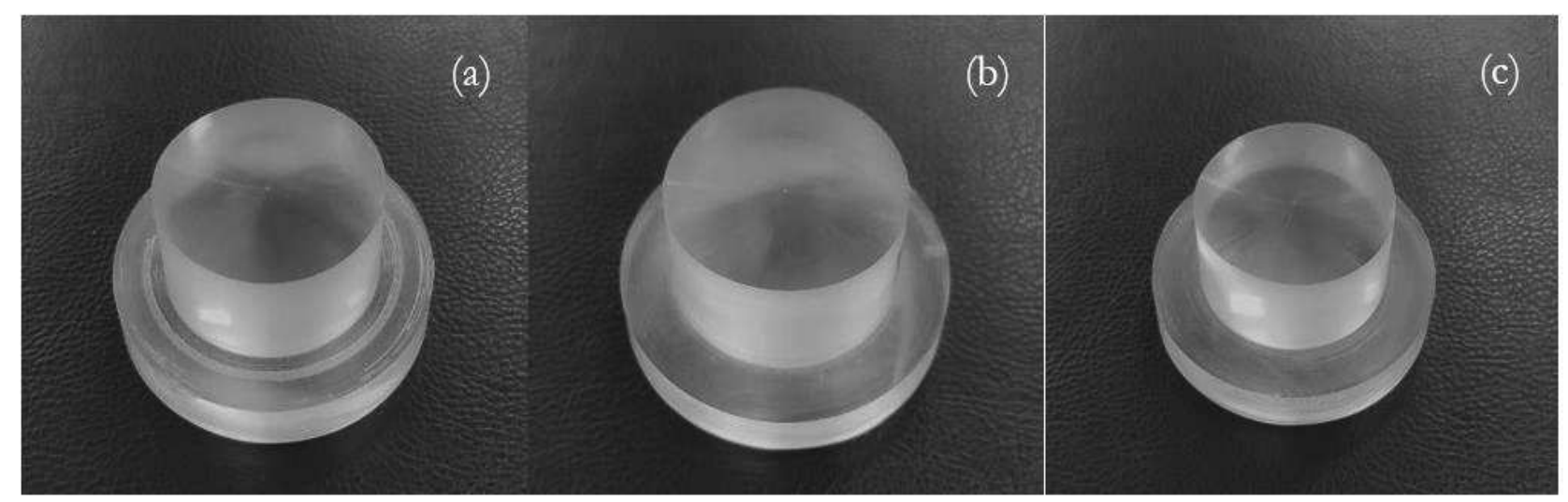

Fig. 18 The macbined workpieces of the toric surface, (a) Workpiece obtained by equal angle discretization method, (b) Workpiece obtained by equal arc length discretization method, (c) Workpiece obtained by equal height discretization method 


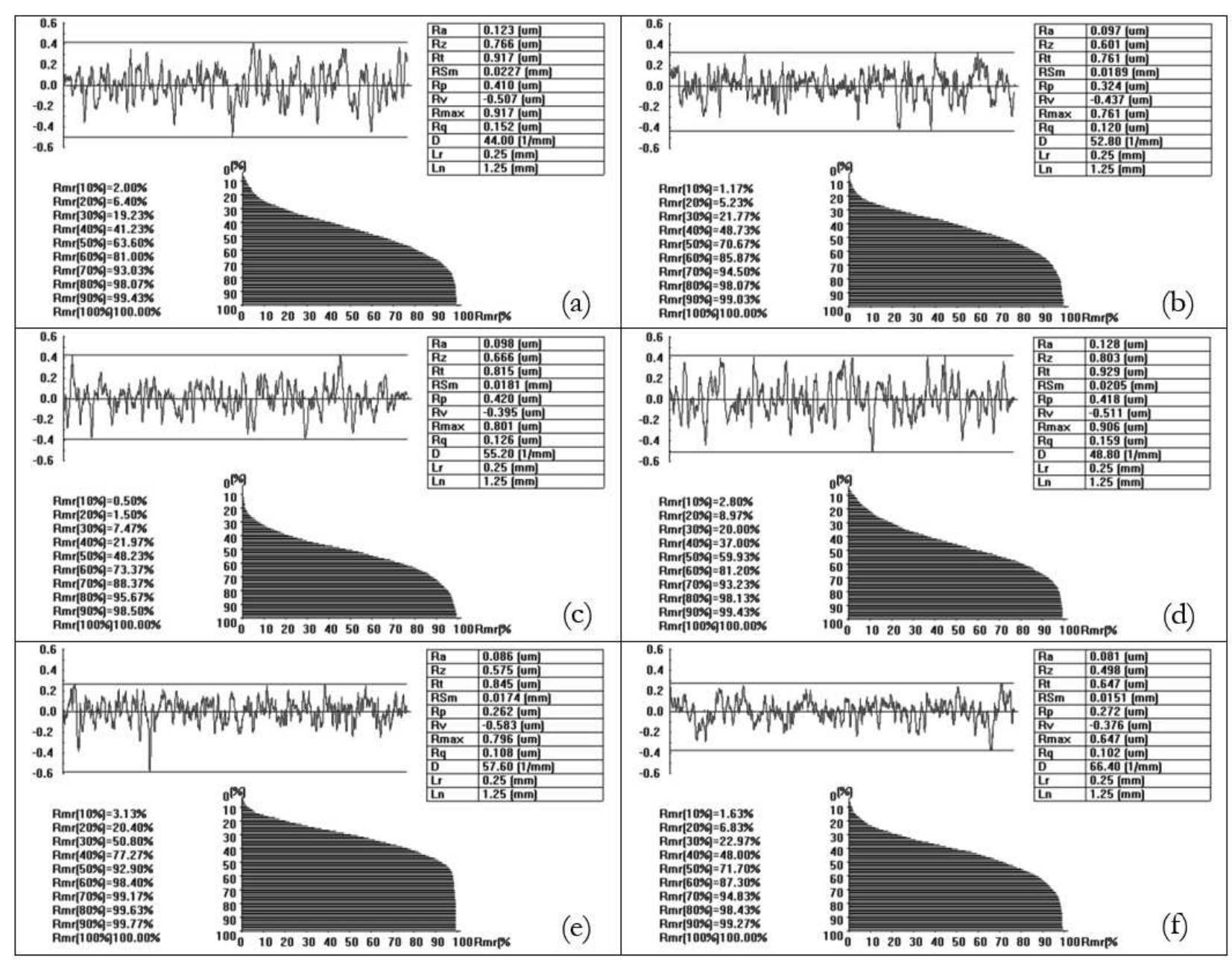

Fig. 19 The measurement result of the surface roughness of the workpieces

(a) The surface roughness value of the outer ring obtained by the equal angle discretization method,

(b) The surface roughness value of the inner ring obtained by the equal angle discretization method,

(c) The surface roughness value of the outer ring obtained by the equal arc length discretization method,

(d) The surface roughness value of the inner ring obtained by the equal arc length discretization method,

(e) The surface roughness value of the outer ring obtained by the equal height discretization method,

(f) The surface roughness value of the inner ring obtained by the equal height discretization method

From Figure 19, it can be seen that the surface roughness value of the outer ring of the workpiece processed by the equal angle discretization method was $0.123 \mu \mathrm{m}$, and the surface roughness value of the inner ring was $0.097 \mu \mathrm{m}$. Therefore, the surface roughness value of the outer ring was greater than that of the inner ring. The main reason is that the discrete points of the outer ring are too sparse, resulting in a larger discrete error. The surface roughness value of the outer ring of the workpiece processed by the equal arc length discretization method was $0.098 \mu \mathrm{m}$, and the surface roughness value of the inner ring was $0.128 \mu \mathrm{m}$. Therefore, the surface roughness value of the inner ring was greater than that of the outer ring. The main reason is that the discrete points of the inner ring are too sparse, resulting in a larger discrete error. The surface roughness value of the outer ring of the workpiece processed by the equal height discretization method was $0.086 \mu \mathrm{m}$, and the surface roughness value of the inner ring was $0.081 \mu \mathrm{m}$. The surface roughness values of the inner ring and the outer ring were basically the same, and both were smaller than the two equal parameter discrete methods. It indicates that the equal height discretization method can effectively reduce the discrete error and improve the processing quality of the workpiece surface.

Figure 20 shows the workpieces of toric surface processed by different interpolation methods using equal height discretization methods. In order to evaluate the form accuracy of the machined workpieces, the surfaces machined were measured by MQ686 coordinate measuring machine. After processing the data, the form error distribution was obtained, as shown in Figure 21. 


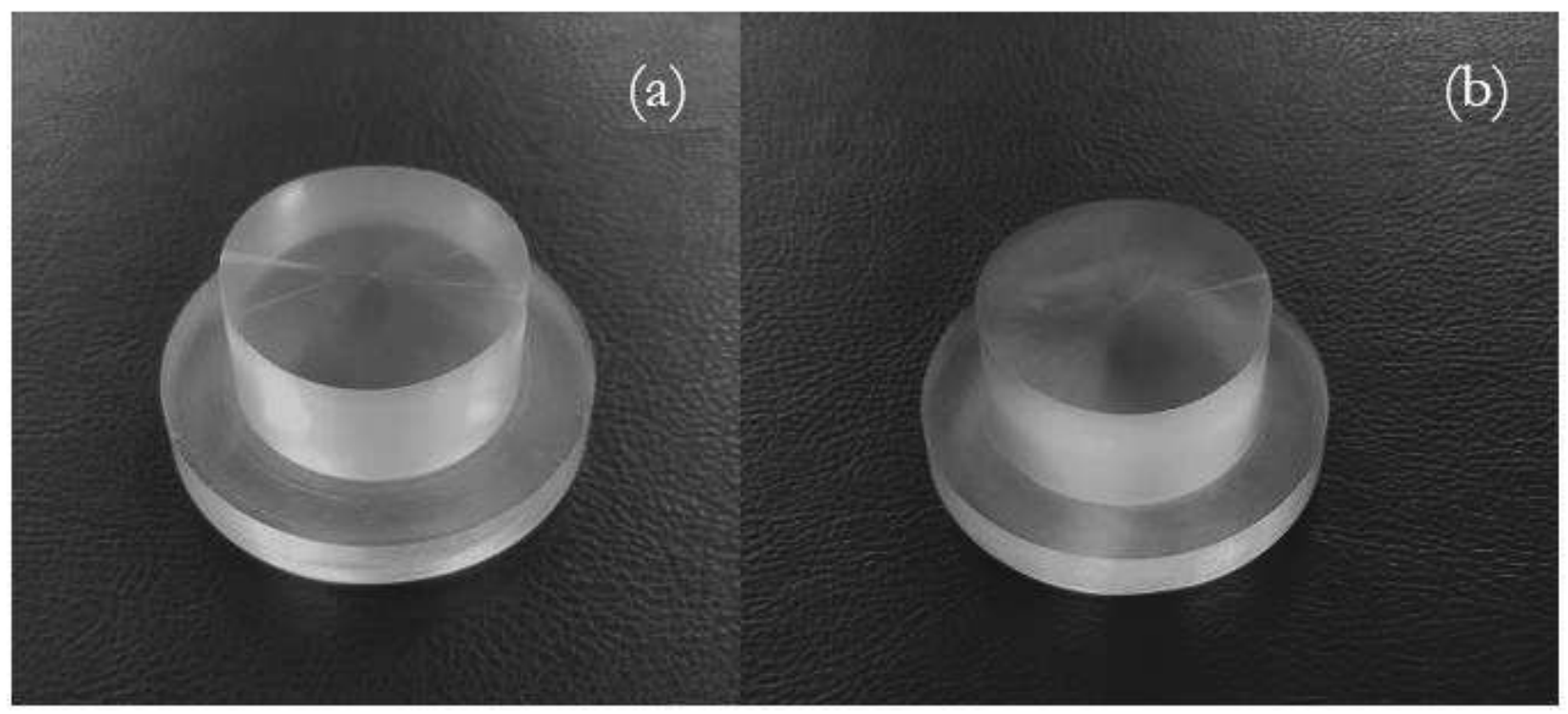

Fig. 20 The workpieces of toric surface processed by different interpolation methods using equal height discretization methods, (a) Workpiece processed by non-uniform node and segment cubic Hermite interpolation, (b) Workpiece processed by non-uniform node and segment cubic spline interpolation

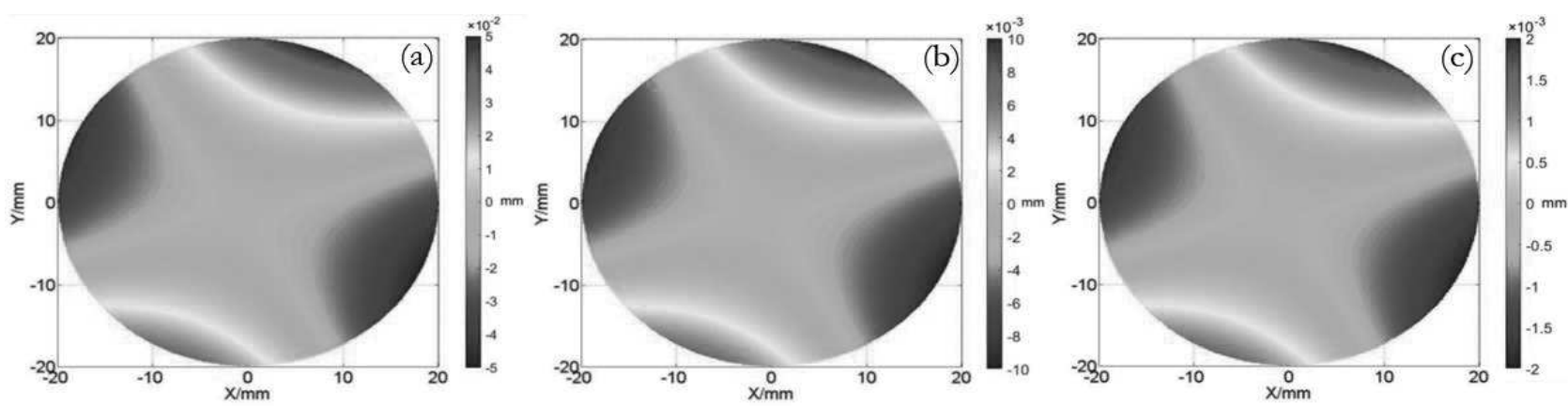

Fig. 21 The form error distribution of the toric surfaces

(a) The form error of the workpiece obtained by the uniform node and segment cubic Hermite interpolation,

(b) The form error of the workpiece obtained by the non-uniform node and segment cubic Hermite interpolation,

(c) The form error of the workpiece obtained by the non-uniform node and segment cubic spline interpolation

From Figure 21, it can be seen that the PV (Peakto-valley) value of the form error of the workpiece obtained by the uniform node and segment cubic Hermite interpolation was about $0.05 \mathrm{~mm}$, the PV value of the form error of the workpiece obtained by the nonuniform node and segment cubic Hermite interpolation was about $0.01 \mathrm{~mm}$, and the PV value of the form error of the workpiece obtained by the non-uniform node and segment cubic spline interpolation was about $0.002 \mathrm{~mm}$. Comparing Figure 21(a) and Figure 21(b), it was observed that the interpolation error could be effectively reduced by the non-uniformization processing, thereby improving the surface accuracy. Comparing Figure 21(b) and Figure 21(c), it was observed that the interpolation error could also be effectively reduced by using the triangle rotary method to convert the segment cubic Hermite interpolation to the segment cubic spline interpolation. As a result, the surface accuracy and the processing quality was improved.

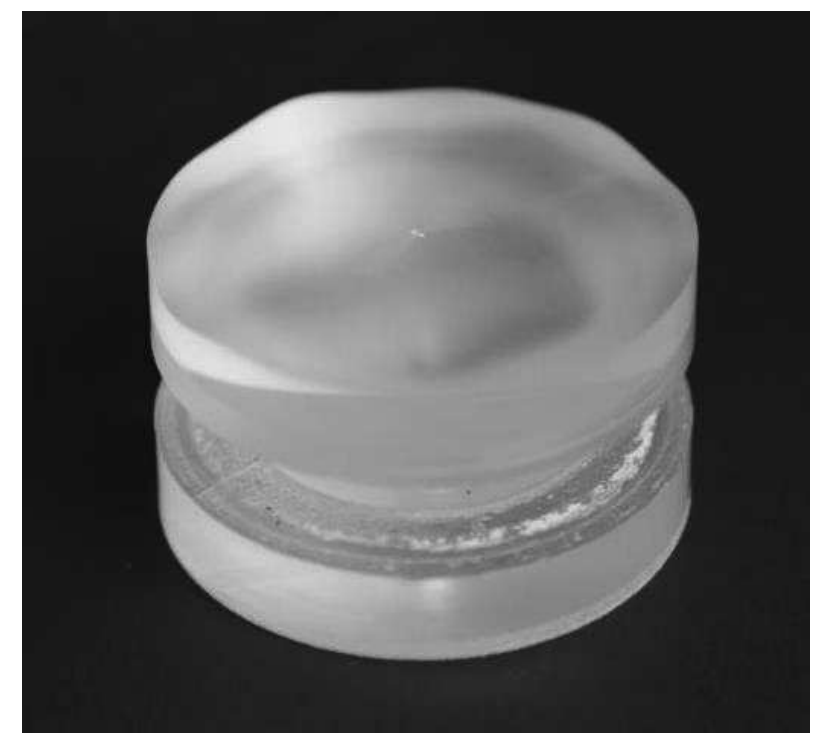

Fig. 22 The machined workpiece of the sinusoidal array surface 
The sinusoidal array surface was machined by using the equal height discretization method and the non-uniform node segment cubic spline interpolation. Figure 22 shows the machined workpiece of the sinusoidal array surface. Figure 23 shows the form error distribution. It can be seen that the PV value of the form error was about $0.009 \mathrm{~mm}$. Figure 24 shows the measurement result of its surface roughness. It can be seen that the value of surface roughness was $0.064 \mu \mathrm{m}$. The case study indicated that the tool path generation method proposed in this paper could be used to fabricate the complex surfaces by STS turning and improve the processing quality.

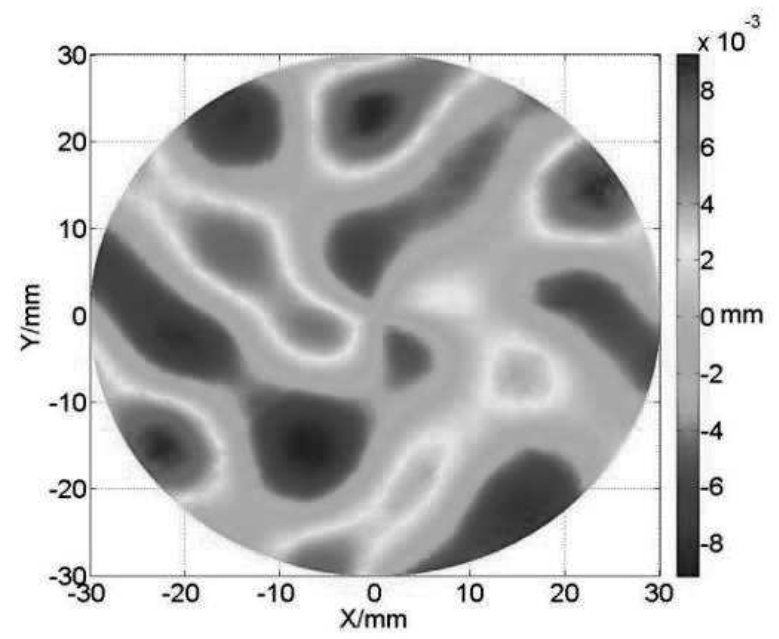

Fig. 23 The form error distribution of the sinusoidal array surface

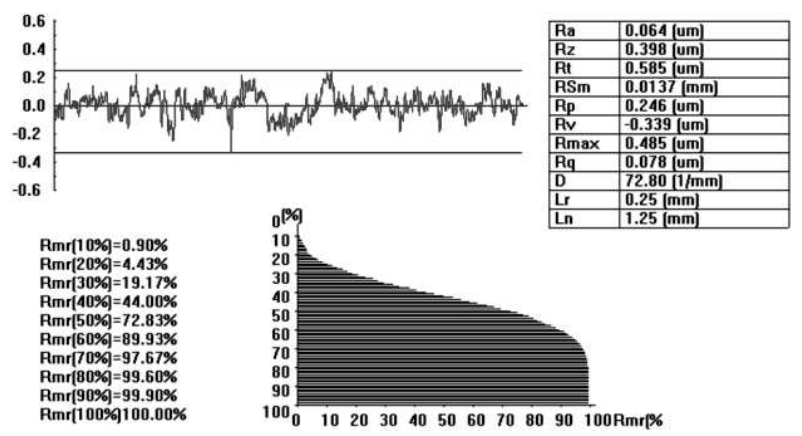

Fig. 24 The measurement result of the surface roughness of the sinusoidal array surface

\section{Conclusions}

The method of the tool path generation for the STS turning of complex surfaces was studied systematically in this paper. Based on the research results, the following conclusions can be drawn:

(1) For the STS turning of complex surfaces, an equal height discretization method was proposed. This method limited the height difference between two adjacent CCPs, thereby the discrete error was reduced, which could reduce the discrete error by more than $70 \%$. In addition, the distribution of the CCPs in the $\mathrm{Z}$ direction obtained by the equal height discretization method was relatively uniform. As a result, the speed and acceleration of the $Z$ axis varied in a small range, and this improved the dynamic performance and machining accuracy.

(2) A new non-uniform node interpolation algorithm was proposed. This method achieved the purpose of non-uniformity processing by increasing the interpolation nodes at the position where the curvature of the trajectory curve is larger thereby reducing the interpolation error, and could reduce the maximum interpolation error by about $70 \%$. In addition, the interpolation error was also reduced by the densification of the interpolation nodes. Furthermore, the simulation analysis results showed that the interpolation error was reduced by two orders of magnitude by transforming segment cubic Hermite interpolation into segment cubic spline interpolation.

(3) With processing experimental research of both sinusoidal array surface and toric surface, the results showed that the value of surface roughness of the toric surface processed by the equal height discretization method is significantly less than that of the equal parameter discretization method. As such, this method could effectively reduce the discrete error and improve the processing quality of the workpiece's surface. The PV value of the form error of the workpiece of the toric surface obtained by non-uniformity processing and segment cubic Spline interpolation reached $0.002 \mathrm{~mm}$, so this method could reduce the interpolation error and improve the surface accuracy effectively. The PV value of the form error of the workpiece of the sinusoidal array surface obtained by non-uniformity processing and segment cubic Spline interpolation was about $0.009 \mathrm{~mm}$, and the value of surface roughness was $0.064 \mu \mathrm{m}$. Therefore, the tool path generation method proposed in this paper can be applied to the STS turning to process complex curved surfaces and can improve the quality of workpiece processing significantly.

\section{References}

[1] NGUYEN VAN TUONG (2019). Advanced CAD/CAM Techniques for 5-Axis Machining of Free-Form Surfaces. In: Manufacturing Technology, Vol. 19, NO 2, pp. 332-336. ISSN

[2] TUONG NGUYEN VAN, NATASA NAPRSTKOVA (2019). Matlab-based Calculation Method for Partitioning a Free-form Surface into Regions. In: Manufacturing Technology, Vol. 19, NO 3, pp. 518-524. ISSN

[3] DAVID DOBROCKÝ (2019). Assessment of Surface Structure of Machined Surfaces. In: Manufacturing Technology, Vol. 19, NO 4, pp. 563572. ISSN 
[4] HUANG YUETIAN (2019). Research on Single Point Diamond Turning Technology for Complex Surface. Ph.D thesis, Chinese Academy of Sciences University, Beijing, China. ISSN

[5] LIU YUNZHU (2012). Design of Equipment and Tool Path Planning of Aspherical Turning. Ph.D thesis, Nanjing Agricultural University, Nanjing, China. ISSN

[6] TIE GUIPENG (2009). Research on the Key Technology of Slow Tool Servo Machining of Free form Optical Elements. Ph.D thesis, $\mathrm{Na}$ tional University of Defense Technology, Changsha, China. ISSN

[7] BAO QIONGQIONG, WANG YU, PAN JU, et al. (2013). Research on Ultra-precision Slow Tool Servo Turning Technology of Free Optical Surface. In: Modern Manufacturing Engineering, Vol. 11, pp. 60-63. China. ISSN

[8] MA FURONG (2018). Free-form Optical Surface Processing in Slowing Tool Turning and Simulation. Ph.D thesis, Lanz̧bou University of Technology, Lanzhou, China. ISSN

[9] WANG XINGSHENG (2014). Research on the Key Technologies of Slow Tool Servo Turning for Complex Optical Surface. Ph.D thesis, Nanjing Agriculture University, Nanjing, China. ISSN

[10] GUAN CHAOLIANG (2010). Study on the Technology of Slow Tool Servo Uitra-precision Diamond Turning for Complex Optical Surface. Ph.D thesis, National University of Defense Technology, Changsha, China. ISSN

[11] GONG H, WANG Y, SONG L, et al. (2015). Spiral Tool Path Generation for Diamond Turning Optical Free-form Surfaces of Quasirevolution. In: Comput Aided Design, Vol. 59, pp. 15-22. China. ISSN

[12] LIU RS (2014). Study of the Interpolation of the Tool Path in Fast Tool Servo Turning. Master degree thesis, Harbin Institute of Techno$\log y$, Harbin, China. ISSN

[13] FANG F Z, ZHANG X D, HU X T (2008). Cylindrical Coordinate Machining of Optical Free-form Surfaces. In: Opt Express, Vol. 16, pp. 7323-7329. United States. ISSN

[14] TIAN F J, YIN Z Q and LI S Y (2015). Fast Tool Servo Diamond Turning of Optical Freeform Surfaces for Rear-view Mirrors. In: Int J Adv Manuf Tech, Vol. 80, pp. 1759-1765. Springer. Germany. ISSN
[15] HSU W Y, LIU Y L, CHENG Y C, et al. (2012). Design, Fabrication, and Metrology of Ultra-precision Optical Free-form Surface for Progressive Addition Lens With B-spline Description. In: Int J Adv Manuf Tech, Vol. 63, pp. 225-233. Springer. Germany. ISSN

[16] CHEN X, KANG M, WANG X S, et al. (2017). Tool Path Optimal Design for Slow Tool Servo Turning of Complex Optical Surface. In: Proceedings of the Institution of Mechanical Engineers Part B-Journal of Engineering Manufacture, Vol. 231, No. 5, pp. 825-837. England. ISSN

[17] ZHANG X D, FANG F Z, WANG H B, et al. (2009). Ultra-precision Machining of Sinusoidal Surfaces Using the Cylindrical Coordinate Method. In: J Micromech Microeng, Vol. 19, pp. 1050-1055. England. ISSN

[18] HU Y, XU M X, XU X Z, et al. (2014). An Accurate Interpolator for FTS Diamond Turning of Optical Free-form Surface. In: Int J Adv Manuf Tech, Vol. 73, pp. 635-638. Springer. Germany. ISSN

[19] WANG X S, KANG M (2012). Cutting Path Planning for Complex Optical Surface Using Hermite Interpolation. In: Journal of Mechanical Engineering, Vol. 48, No. 11, pp. 191-198. China. ISSN

[20] CHI W S, TANG S J (2009). Analysis and Application of PVT Mode of PMAC Motion Controller. In: Macbinery, Vol. 7, pp. 35-37. China. ISSN

[21] LI Q Y, WANG N C, YI D Y (2008). Numerical Analysis, pp. 22-312. Tsinghua University Publisher, China. ISBN

[22] XU X Y, ZHONG T Y (2006). Construction and Realization of Cubic Spline Interpolation Function. In: Ordnance Industry Automation, Vol. 25, No. 11, pp. 76-78. China. ISSN

[23] WANG X S, KANG M (2012). Toric Spectacle Lens by Using Slow Tool Servo Turning. In: China Mechanical Engineering, Vol. 18, No. 23, pp. 2167-2173. China. ISSN

[24] YU H J (2015). Theoretical and Technological Research on Optical Free-form Surface of Single Point Diamond Ultra-precision Turning. Master degree thesis, Jilin university, Jilin, China. ISSN 